\title{
Characterizing divergent spin-orbit coupling effects on ultrafast nonradiative decay in transition-metal compounds
}

\author{
William P. Carbery and Daniel B. Turner \\ Department of Chemistry, New York University, 100 Washington Square East, New York NY 10003 USA
}

\begin{abstract}
Two-dimensional electronic spectroscopy reveals divergent, spinorbit coupling mediated, electronic relaxation dynamics in iridium(IV) hexabromide $\left(\left[\mathrm{IrBr}_{6}\right]^{2-}\right)$ and the ruthenium(II)-based DSSC dye N719.
\end{abstract}

\section{Introduction}

Conventional descriptions of excited state relaxation involve three decay pathways locked in a rigid temporal hierarchy. Best summarized by Kasha's rule, in which fluorescence always proceeds from the lowest energy excited state, this description states that molecules first undergo vibrational relaxation followed by internal conversion followed by intersystem crossing [1]. Ultrafast laser spectroscopy measurements conducted over the last three decades suggest that this hierarchy is far from accurate. Measurments on rhodopsin, for instance, revealed that conical intersections can lead to internal conversion rates that are on the same timescales as vibrational relaxation [2]. Subsequent computational work suggested that conical intersections can act as mediators during photochemical reactions and exist as important bottlenecks in far more systems than previously thought possible [3].

Although internal conversion mediated by conical intersections is now considered ubiquitous, intersystem crossing is still usually considered slower than other competing relaxation pathways. Nevertheless, early transient absorption measurements on $\left[\mathrm{Ru}(\mathrm{bpy})_{3}\right]^{2+}$ demonstrated intersystem crossing as fast as 150 fs [4]. Similar experiments on the iron-containing variant, which is a spin-crossover system, also found an ultrafast intersystem crossing rate [5]. Detailed analysis concluded that the rate of intersystem crossing was independent of the magnitude of spin-orbit coupling, presumably the mechanism for ultrafast conversion between states of differing spin-multiplicity. Instead, a threshold value determines if intersystem crossing competes with other relaxation mechanisms, as demonstrated by the measurements of some Pt, Pd, Ni, Os, and Re containing compounds [6-8].
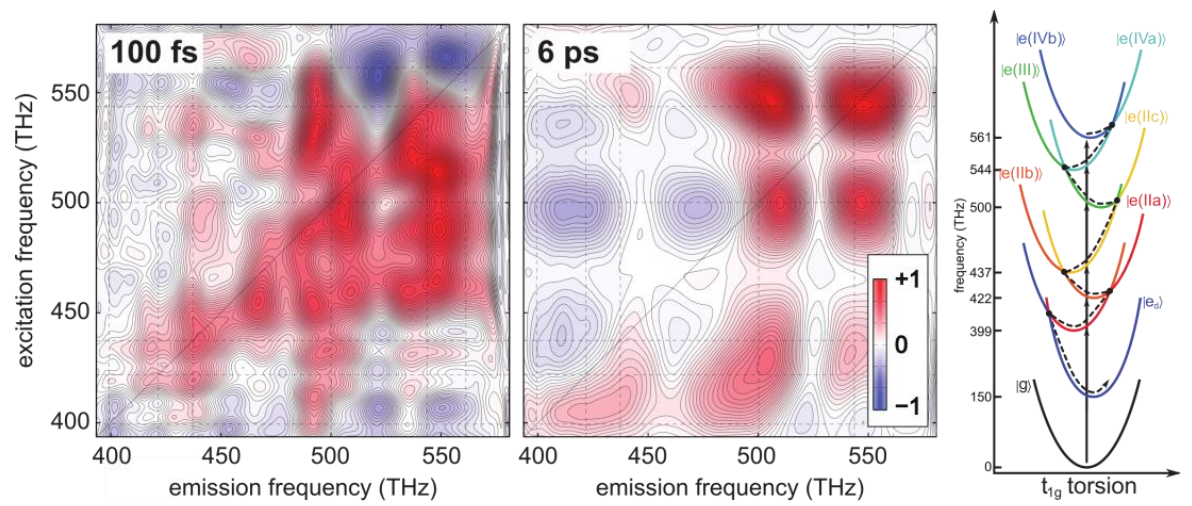

Fig. 1. The 2D electronic spectra of $\left[\mathrm{IrBr}_{6}\right]^{2-}$ at waiting times of $100 \mathrm{fs}$ and $6 \mathrm{ps}$ (left). A comparison reveals the rapid loss of ESA signal at high excitation frequencies concurrent with the conversion of stimulated emission signal into ESA signal in the $390-460 \mathrm{THz}$ emission range. The schematic representation (right) was constructed using a simple group theory model in the total angular momentum basis. This figure has been adapted from ref. 13 .

Much like the early work on internal conversion and conical intersections, recent experiments on intersystem crossing suggest that transitions between two potential energy surfaces of differing spin multiplicity are highly nonadiabatic [9]. The fundamental question remains to what degree intersystem crossing is beyond the BornOppenheimer approximation, and what specific combination of spin-orbit coupling, surface topology, and local geometry is likely to yield ultrafast decay [10]. The best molecular targets for resolving these questions involve transition-metal compounds, which easily exceed the spin-orbit coupling threshold established by earlier experiments. However, ultrabroadband absorption spectra and low molar absorptivities make transition-metal compounds exceptionally difficult to measure and study. To address these challenges, we used high-sensitivity

*Corresponding author: dturner@nyu.edu 
two-dimensional electronic spectroscopy (2D ES) to measure iridium and ruthenium molecules. These spectra reveal two different spin-orbit coupling effects in the ultrafast relaxation of transition-metal compounds.

\section{Results and Discussion}

Our group has developed a robust ultrabroadband, high-sensitivity, passively phase stable two-dimensional electronic spectrometer with minimal directional-filter and phase-mismatch distortions $[11,12]$. The 2D spectra of $\left[\mathrm{IrBr}_{6}\right]^{2-}$ at $100 \mathrm{fs}$ and $6 \mathrm{ps}$ are presented in Fig. (1). The complete coupling of all LMCT bands in the chromophore produces several dozen individual peaks, and the stimulated emission signal in the low frequency, $390-460 \mathrm{THz}$, range of the $100 \mathrm{fs}$ spectrum rapidly converts to excited state absorption (ESA) signal. Indeed, the ultrafast dynamics of $\left[\mathrm{IrBr}_{6}\right]^{2-}$ are best explained as interactions between spin-orbit coupled, adiabatic potential energy surfaces resulting in nonradiative decay of all LMCT transitions into the lowest excited state [13]. The observation of persistent vibrational coherence during this LMCT energy relaxation suggested conical intersection mediated nonradiative decay. This necessitated expanding the conical intersection definition to include a crossing between states of the same total angular momentum rather than merely the same spinmultiplicity [14]. This more general definition of a conical intersection helps to understand other, unusual aspects of spin-orbit coupled systems, including the observation of ultrafast intersystem crossing among the LMCT bands of $\left[\operatorname{IrBr}_{6}\right]^{2-}$, but not between its lowest-lying excited state and ground state. Indeed, original work with $\left[\mathrm{IrBr}_{6}\right]^{2-}$ even drew comparisons with the Jahn-Teller distortion present in $\left[\mathrm{CuCl}_{4}\right]^{2-}$, itself a special conical intersection but in a system with spin-orbit coupling likely above the threshold needed for ultrafast intersystem crossing [15].
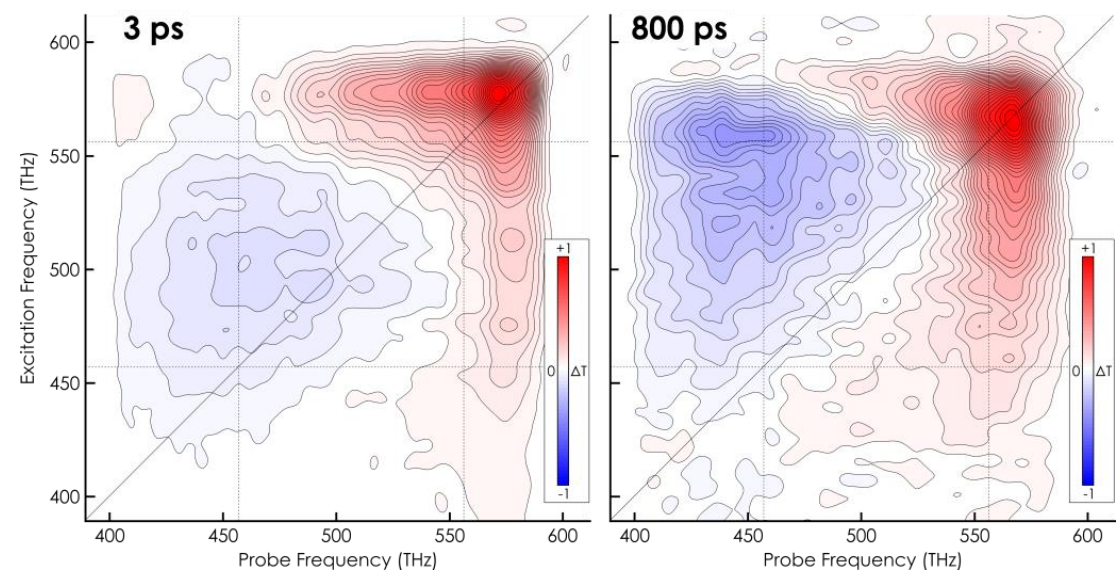

Fig. 2. The 2D electronic spectra of the ruthenium-based DSSC dye, N719, at waiting times of 3 ps and 800 ps. The dominant ESA feature at probe frequencies less than $500 \mathrm{THz}$ shifts from the initially prepared state at $500 \mathrm{THz}$ excitation to the excitation maximum of N719 as an approximately 20 ps transient species nonradiatively decays.

Subsequent 2D ES measurements on the N719 dye molecule $\left(\left[\mathrm{Ru}(\mathrm{N}-\mathrm{N})_{2}(\mathrm{NCS})_{2}\right]^{2+}\right.$, where $(\mathrm{N}-\mathrm{N})$ is the bidentate, singly deprotonated 2,2'-bipyridyl-4,4'-dicarboxylato ligand) reveal more conventional spectral features and dynamics. At a waiting time of 3 ps, the 2D spectrum in Fig. (2) contains weak stimulated emission features relative to the dominant ESA and bleach peaks with vibronic progressions. Even at high excitation frequencies, where the ESA feature is weaker than the bleach signal, stimulated emission from the ${ }^{1}$ MLCT bands appears weak, which is consistent with prior reports of ultrafast nonradiative decay to a ${ }^{3}$ MLCT manifold through intersystem crossing [16]. The changes in the N719 spectra over time are significantly slower and subtler than the changes in the $\left[\mathrm{IrBr}_{6}\right]^{2-}$ spectra, with the most prominent change the shift in ESA excitation frequency from $500 \mathrm{THz}$ at $3 \mathrm{ps}$ to the excitation maximum of N719 $(553 \mathrm{THz})$ at $800 \mathrm{ps}$. Analysis of the transient absorption data suggests that this spectral shift is the result of an approximately 20 ps disappearance of transient signal that suppresses bleach features and leads to excitation dependent growth in the ESA region.

The relatively long-lived transient species is inconsistent with either solvation effects or relaxation into the triplet manifold, which is generally considered to be ultrafast [4]. Indeed, previous observations on a class of $\mathrm{Ru}(\mathrm{II})$ dyes suggest that local symmetry changes can affect the picosecond kinetics of the molecule, as intersystem crossing and internal conversion compete nonradiatively [17]. The analysis of the $\left[\operatorname{IrBr}_{6}\right]^{2-}$ data suggests that spin-orbit coupling has the potential to account for these local symmetry changes, especially if these nonradiative pathways are more metal-centered than the charge transfer states. A similar process, where the spatial symmetry of the d-orbitals is altered but the structural geometry of the system is preserved, could explain the coexistence of fast MLCT transitions with slow nonradiative decay. In that case, the 2D spectra of N719 at 
early time reflects complete relaxation into the ${ }^{3} \mathrm{MLCT}$ manifold but incomplete relaxation of the metal-centered excited states. At sufficiently long waiting times the metal-centered states have relaxed into the lowest lying state of complementary angular momentum and, without the overlapping transient signal, the ESA is prominently featured on the excitation line of N719.

Spin-orbit coupling is clearly an important mediator of transition metal compound ultrafast dynamics. However, the divergent effects of this coupling on two dye molecules, and the vastly different kinetics of their relaxation processes, requires more experimental investigation. Determining how spin-orbit coupling produces and modifies the competing interactions of ultrafast internal conversion, intersystem crossing, and vibrational relaxation also requires the excitation specificity and temporal resolution of 2D ES. We expect that continued improvements of 2D ES will lead to better insights into the ultrafast dynamics of both transition-metal compounds and spin-orbit coupling.

\section{References}

[1] M. Kasha, "Characterization of electronic transitions in complex molecules," Discuss. Faraday. Soc. 914 (1950).

[2] Q. Wang, R. W. Schoenlein,L. A. Peteanu, R. A. Mathies, and C. V. Shank, "Vibrationally coherent photochemistry in the femtosecond primary event of vision," Science. 266, 422 (1994).

[3] M. A. Robb, "In this molecule there must be a conical intersection,” Advances in Physical Organic Chemistry. 48, 189 (2014).

[4] N. H. Damrauer, G. Cerullo, A. Yeh, T. R. Boussie, C. V. Shank, and J. K. McCusker, "Femtosecond dynamics of excited-state evolution in $\left[\mathrm{Ru}(\mathrm{bpy})_{3}\right]^{2+}$," Science. 275, 54 (1997).

[5] W. Gawelda, A. Cannizzo, V-T. Pham, F. van Mourik, C. Bressler, and M. Chergui, "Ultrafast nonadiabatic dynamics of [Fe $\left.{ }^{\text {II }}(\text { bpy })_{3}\right]^{2+}$ in solution,” J. Am. Chem. Soc. 129, 8199 (2007).

[6] C. Sousa, C. de Graaf, A. Rudavskyi, R. Broer, J. Tatchen, M. Etinski, and C. M. Marian, "Ultrafast deactivation mechanism of the excited singlet in the light-induced spin crossover of $\left[\mathrm{Fe}(2,2 \text { '-bipyridine })_{3}\right]^{2+}$," Chem. Eur. J. 19, 17541 (2013).

[7] F. Frei, A. Rondi, D. Espa, M. L. Mercuri, L. Oilia, A. Serpe, A. Odeh, F. Van Mourik, M. Cherguim T, Feurer, P. Deplano, A. Vlcek Jr., and A. Cannizzo, "Ultrafast electronic and vibrational relaxations in mixed-ligand dithione-dithiolato Ni, Pd, and Pt complexes," Dalton Trans. 43, $17666(2014)$.

[8] A. Cannizzo, A. M. Blanco-Rodriquez, A. El Nahhas, J. Sebera, S. Zalis, A. Vlcek Jr., and M. Chergui, "Femtosecond fluorescence and intersystem crossing in rhenium(I) carbonyl-bipyridine complexes," J. Am. Chem. Soc. 130, 8967 (2008).

[9] M. Chergui, "Ultrafast photophysics of transition metal complexes,” Acc. Chem. Res. 48, 801 (2015).

[10] M. A. El-Sayed, "The triplet state: its radiative and nonradiative properties," Acc. Chem. Res. 1, 8 (1968).

[11] L. A. Bizimana, J. Brazard, W. P. Carbery, T. Gellen, and D. B. Turner, "Resolving molecular vibronic structure using high-sensitivity two-dimensional electronic spectroscopy,” J. Chem. Phys. 143, 164203 (2015).

[12] M. K. Yetzbacher and D. Jonas, "Propogation, beam geometry, and detection distortions of peak shapes in two-dimensional Fourier transform spectra," Chem. Phys. 126, 044511 (2007).

[13] W. P. Carbery, A. Verma, and D. B. Turner, "Spin-orbit coupling drives femtosecond nonadiabatic dynamics in a transition metal compounds,” J. Phys. Chem. Lett. 8, 1315 (2017).

[14] F. Bernardi, M. Olivucci, and M. A. Robb, "Potential energy surface crossings in organic photochemistry," Chem. Soc. Rev. 25, 321 (1996).

[15] S. M. Matveev, A. S. Mereshchenko, M. S. Panov, and A. N. Tarnovsky, "Probing the fate lowest-energy near-infrared metal-centered electronic excited states: CuCl42- and IrBr62-," J. Phys. Chem. B. 119, 4857 (2015).

[16] S. E. Koops, B. C O'Regan, P. R. F. Barnes, and J. R. Durrant, "Parameters influencing the efficiency of electron injection in dyesensitized solar cells," J. Am. Chem. Soc. 131, 4808 (2009).

[17] Wächtler, M.; Maiuri, M.; Brida, D.; Popp, J.; Rau, S.; Cerullo, G.; Dietzek, B. "Utilizing Ancillary Ligands to Optimize the Photophysical Properties of 4H-Imidazole Ruthenium Dyes”. Chem. Phys. Chem. 14, 2973 (2013). 